\title{
Cambios respiratorios post-abdominoplastia
}

\section{Postahdominoplasty respiratory changes}

\author{
Vaca Escobar, B.*, Guerra Navarrete, D.*, Manzano Manzano, R.**
}

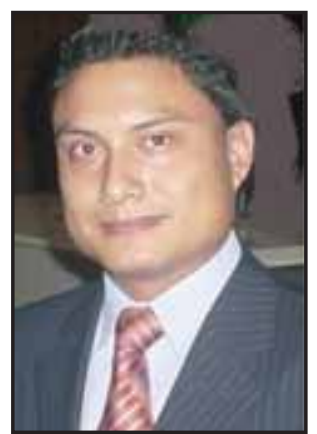

Vaca Escobar, B.
Resumen

El contorno corporal ha sido preocupación para la cirugía plástica desde hace muchas décadas. Sin embargo, cirugías como la abdominoplastia no están exentas de complicaciones a nivel respiratorio, como las resultantes de la plicatura abdominal.

Nuestro estudio se basa en la investigación de estos cambios post-abdominoplastia y en si después de 15 días, los mismos regresan a sus valores iniciales. Evaluamos 18 pacientes de sexo femenino con abdómenes péndulos grado II y III de Matarasso sin antecedentes de problemas respiratorios, a las que se les realizó abdominoplastia clásica en todos los casos, con una plicatura de rectos abdominales de $8.54 \mathrm{~cm}$. en promedio, con capacidad vital preoperatoria media de 2,88 (97\%) en el postoperatorio inmediato de $2,52(77.5 \%)$ y a los quince días de $2.73(86.6 \%)$. Se encontró una diferencia estadística entre los valores de capacidad vital pre y postoperatoria y una recuperación parcial (50\%) del valor basal a los 15 días. Concluimos que nuestro estudio refuerza lo referido por otros autores en cuanto a la variación de la presión intrabdominal pre y postplicatura y su influencia en la capacidad vital pulmonar; además demostramos que ésta se recupera sólo parcialmente hasta sus valores iniciales una vez transcurridos 15 días desde el procedimiento quirúrgico.

\begin{tabular}{|c|c|}
\hline Palabras clave & $\begin{array}{l}\text { Abdominoplastia. Cambios respirato- } \\
\text { rios. }\end{array}$ \\
\hline Código numério & 311 \\
\hline
\end{tabular}

The body contour has been one of the main concerns for plastic surgery for many decades. However, surgical procedures like abdominoplasty are not complication free as a result of increased abdominal pressure over the respiratory tract.

Our study is based on investigating these changes postabdominoplasty and their return to normal after 15 days. We evaluated 18 female patients with abdominal deformity graded II and III according to the Matarasso scale, without history of respiratory problems, who received a classic abdominoplasty without lipoaspiration, with a mean value of the rectus abdominalis plicature of $8.54 \mathrm{~cm}$, and a preoperatory vital capacity of $2,88(97 \%)$, immediate postoperatory value of $2,52(77.5 \%)$, and after 15 days of 2,73 $(86.6 \%)$. We found statistical difference between pre and postoperative values and a partial recovery $(50 \%)$ of the baseline values after 15 days. The conclusions we obtained reinforce the reports made by others authors concerning variations in the abdominal pressure pre and post abdominal surgery, and we found a partial recovery after 15 days after the procedure.

Key words Abdominoplasty. Respiratory Changes.

Numeral Code 5311 


\section{Introducción}

El contorno corporal ha sido la preocupación de las mujeres desde hace mucho tiempo; desde la edad media cuando se usaban ajustados corsés para afinar y modelar la figura, hasta nuestros tiempos en donde los grandes avances en Cirugía Plástica hacen posibles grandes cambios de la silueta corporal.

Fue Voloir, un cirujano general, quien describió en Francia en su Tesis una resección de grasa y piel a nivel abdominal realizada por sus colegas también franceses De Mars y Marx, siendo ésta la primera descripción de este tipo de procedimiento (1). Vinieron después avances sobre todo con respecto a las incisiones, con la incisión elíptica de Morestin en 1911 y la incisión vertical de Schepelman en 1918. Grandes aportes como los de Regnault, Pitanguy en 1974, Baroudi en 1975 y el gran descubrimiento de la Liposucción como complemento por los profesores Illoux y Kesserling, son los que han hecho de este procedimiento uno de los más usados en Cirugía Plástica (2-4).

En cuanto a la relación de la Abdominoplastia con cambios respiratorios, tenemos observaciones realizadas por primera vez por los profesores Pitanguy, Grazer y Goldwin en 1977, quienes reportan que el 0.7\% de sus pacientes presentaron embolia pulmonar, con un $0.002 \%$ de mortalidad de los mismos (6). Ubiglia y Pastacaldi son los primeros en encontrar cambios en la función respiratoria (7) y Yasbeck en México encuentra una relación estrecha entre la capacidad vital pulmonar, la presión intrabdominal y el tamaño de la plicatura; además informan sobre la influencia del cierre de la piel en la elevación de la presión (8).

En los estudios previamente mencionados se demuestra la disminución de la capacidad vital en el postoperatorio hasta en un 25\% (9-14); sin embargo, no tenemos evidencias del tiempo necesario para la recuperación de la misma si es que lo hace. En nuestro estudio intentamos demostrar que la capacidad vital se recupera hasta alcanzar sus valores iniciales, para lo cual hemos tomado como tiempo de corte 15 días post-cirugía, tiempo en el cual evidenciamos mejoría clínica en la capacidad vital de las pacientes en estudio.

\section{Material y método}

Se tomó como universo de estudio a mujeres de entre 35 y 55 años de edad, tributarias de la Seguridad Social, residentes en Quito (Ecuador), sin antecedentes pulmonares y no fumadoras, con abdómenes péndulos tipo II y III según la clasificación de Matarasso (15-17), con paridad satisfecha y con un índice de masa corporal (IMC) de más de 25 , sin hernias, quie- nes aprobaron un test psicológico para desordenes dismórficos y firmaron una hoja de consentimiento informado para el estudio.

\section{Parámetros de evaluación}

\section{Número de hijos}

Tamaño de la plicatura de los rectos: medida en centímetros durante el transoperatorio $(18,19)$.

Presión intrabdominal pre y postoperatoria: tomada indirectamente a través de la medición de la presión intravesical mediante el uso de una sonda colocada previo vaciamiento de la vejiga, tras lo cual se añaden $100 \mathrm{cc}$ de solución salina estéril y se mide la presión en $\mathrm{cm}$. de agua con una regla de presión venosa central convencional, colocando el punto 0 a nivel de la sínfisis del pubis (20).

Espirometria pre, postoperatoria y a los 15 días: este examen fue realizado por el Servicio de Neumología con parámetros establecidos internacionalmente.

A las pacientes se les administraron analgésicos mediante infusión continua y en ciertos casos a través de un catéter epidural, durante las primeras 24 horas postcirugía, con el objeto de eliminar el factor dolor como distractor, sobre todo durante la espirometría realizada a las 24 horas.

Todos los datos obtenidos fueron sometidos a análisis estadístico utilizando el programa Excel®, con el cual se sacaron promedios para luego compararlos mediante T de Students y obtener datos que nos permitieran saber si las diferencias obtenidas fueron o no significativas, con un intervalo de confianza correspondiente al $99 \%$.

\section{Resultados}

Se seleccionaron al final 18 pacientes que cumplieron con los criterios de inclusión para el estudio. Estas pacientes tenían una edad promedio de 34.1 años; número de hijos promedio de 2.4; índice de masa corporal promedio de 27.5. Todas tenían un abdomen péndulo grado III de Matarasso. El promedio de plicatura de los rectos abdominales fue de $8,64 \mathrm{~cm}$., presión intrabdominal prequirúrgica de 4,46 y postoperatoria de 13,56 , encontrándose diferencia significativa entre las dos (Fig.1).

Los resultados de las espirometrías realizadas antes de la cirugía, postcirugía y a los 15 días se encuentran resumidos en la Tabla I.

Al realizar el análisis con T de Students de los valores de capacidad vital pre y postoperatorios se encontró un valor de $\mathrm{p}=0,0004705$, lo que demuestra significancia estadística. No así los valores entre capacidad vital postquirurgica y quince días después de la intervención, en los que se obtuvo un valor de $p=0,0244$ 
que no demuestra una significancia estadística con un grado de confianza de 99\%; sin embargo, tomando en cuenta el porcentaje de recuperación de la capacidad vital, se observó que la disminución del $20.11 \%$ encontrada después de la cirugía se recupera en un 10\% a los 15 días de la misma (Fig 2).

\begin{tabular}{|lccc|}
\hline \multicolumn{4}{|c|}{ Tabla I. Resultados de la espirometría } \\
& Capacidad vital & FEV 1 & FEMM \\
Preoperatorio & $2.88 ; 97 \%$ & $2.57 ; 87.7 \%$ & $3.23 ; 89.5 \%$ \\
Postoperatorio & $2.52 ; 77.5 \%$ & $2.10 ; 86 \%$ & $2.14 ; 89.2 \%$ \\
$\begin{array}{l}\text { Postoperatorio } \\
\text { a los 15 días }\end{array}$ & $2.73 ; 86.6 \%$ & $2.40 ; 86.3 \%$ & $3.30 ; 88.3 \%$ \\
\hline
\end{tabular}

\section{Discusión}

Observamos una diferencia estadísticamente significativa entre los valores de las presiones intrabdominales pre y postoperatorias, por lo que queda comprobado en nuestra serie que la plicatura de rectos abdominales desde $8 \mathrm{~cm}$. en adelante produce aumento importante de la presión intrabdominal y que ésta, al subir el nivel del diafragma, causa una disminución en la capacidad vital pulmonar estadísticamente significativa aunque no en la misma proporción que la elevación de la presión intrabdominal. Estos resultados se correlacionan con lo encontrado por diferentes autores (8-14, 21-23).

En cambio, los resultados a los quince días, no fueron estadísticamente significativos en relación a la capacidad vital, lo que demuestra que si bien es cierto que se recupera la capacidad vital en este lapso de tiempo, esta recuperación no llega hasta los niveles basales iniciales, sino a valores porcentuales correspondientes al 50\% de la misma. Sería interesante probar en un futuro la capacidad pulmonar vital en un periodo más largo de tiempo.

Los valores espirométricos espiratorios como el FEV1 y el FEMM no se modificaron en vista de que no se ven influidos directamente por la presión intrabdominal, como lo demuestra también la literatura consultada (8-11).

\section{Conclusiones}

La dermolipectomía con plicatura de los músculos rectos abdominales influye en la presión intrabdominal elevándola, y ésta a su vez causa importante disminución de la capacidad vital pulmonar, aunque estos valores no son proporcionales entre sí.

La capacidad vital disminuida por el proceso quirúrgico a los 15 días no se llega a recuperar hasta los valores iniciales, aunque sí se recupera parcialmente (50\%).

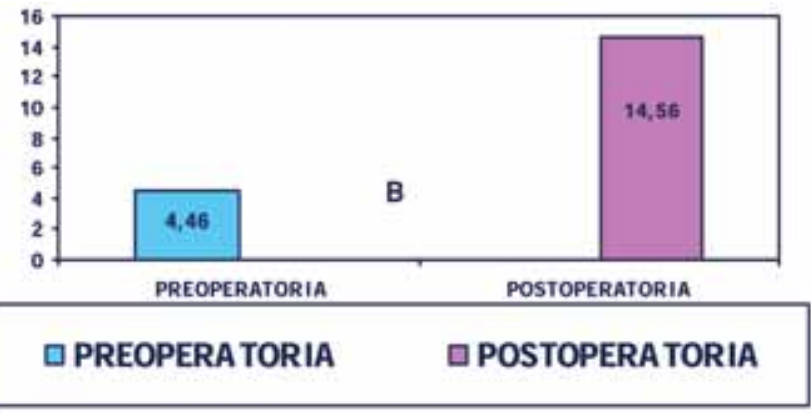

Fig. 1. Presión intraabdominal

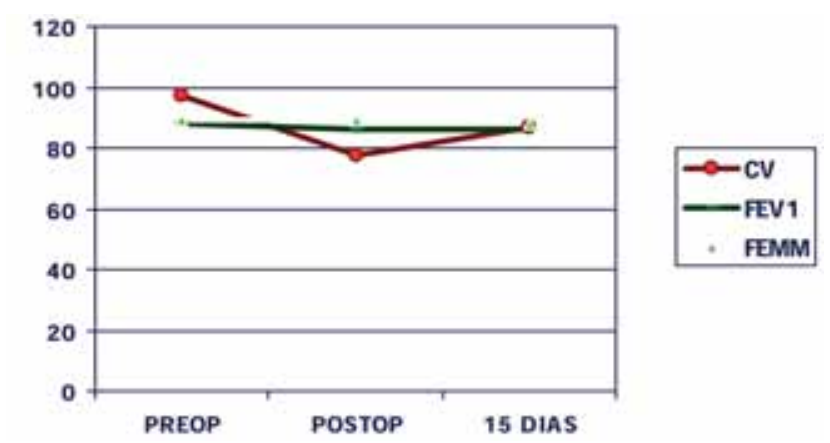

Fig. 2. Curva comparativa de los resultados de la espirometría

Estos resultados nos ayudan a conocer las implicaciones que tiene la dermolipectomía como procedimiento quirúrgico sobre la fisiología pulmonar de nuestros pacientes, ya que debemos tener cuidado en la selección de los mismos para evitar problemas graves.

Observamos que pacientes como las de nuestro estudio, con valores de capacidad vital normales, presentan disminución de hasta el $25 \%$ tras la cirugía; esto nos hace pensar que entonces con pacientes que empezarían con un valor basal de capacidad vital más bajo de lo normal (pacientes con EPOC o fumadoras), tras el procedimiento quirúrgico podrían llegar a valores tan bajos como para causar implicaciones fisiológicas.

Basándonos en estos resultados, creemos conveniente que el cirujano plástico realice siempre ante la sospecha de enfermedad pulmonar una espirometría para asesoramiento neumológico a los pacientes que vayan a ser sometidos a estos procedimientos, con lo cual podríamos anticiparnos mediante la realización de fisioterapia respiratoria en el postoperatorio inmediato, además de educar sobre la realización de ejercicios destinados a aumentar la capacidad vital del acto quirúrgico, para que el organismo se vaya acostumbrando a los cambios que se producirán con la cirugía.

Dirección del autor

Dr. Byron Vaca

e-mail: byronvaca@hotmail.com 


\section{Bibliografía}

1. Hunter GR, Crapo RO.: "Pulmonary complications following abdominal lipectomy”. Plast. Recons. Surg. 1983;3:71.

2. Obeid F, Fath AS.: "Increases in intrabdominal pressure effect pulmonary compliance”. Arch. Surg. 1995;130:544.

3. Pitanguy I.: "Abdominal lipectomy an approach to it through an analysis of 300 consecutive cases". Plast. Reconst. Surg. 1967;40:384.

4. Rizzo A. Davis PC.: "Intraoperative vesical pressure measurements as a guide in the closure of abdominal wall defects". J. Am. Coll. Surg . 1996;62:1992.

5. Bozzola AR. Psilliakis JM.: "Abdominoplasty. A new concept and classifications treatment”. Plast. Reconstr. Surg. 1988;82: 983.

6. Matarasso A.: "Abdominoplasty". Clin. Plast. Surg. 1998;16(2):289.

7. Ubiglia GP. Pastacaldi P.: "Nuovo metodo di abdominoplastica estetica”. Riv.Ital.Chir.Plast 1977;9:257.

8. Nahas FX. Augusto SM.: "Should diastasis recti be corrected?" Aesth. Plast. Surg. 1997;21:285.

9. Marin JA. Saucedo JA.: "Variation of intrabdominal pressure caused by abdominoplasty in healthy woman". Rev. Gastroenterol. Mex. 2004;69(3):156

10. Al-Basti Habib B. El-Khatib, Hamdy A. Taha, Ahmed Sattar, Hisham Bener.: "Intrabdominal pressure after full abdominoplasty in obese multiparous patients". Plast Reconst. Surg. 2004;113(7):2145.

11. Al-Qattam MM.: "Abdominoplasty in multiparous women with severe musculoaponeurotic laxity”. Br.J.Plast.Surg. 1997;50(6) $: 450$.
12. Talisman R, Kaplan B, Haik J, Aronov S, Shraga A, Orenstein A.: "Measuring alterations in intrabdominal pressure during abdominoplasty as a predictive value for possible postoperative complications". Aesth. Plast. Surg. 2002; 26(3):189.

13. Yasbeck R. López S.: "Cambios en la presión intrabdominal en la función respiratoria post-abdominoplastia”. Cir. Plástica. 2003;13 (2):56.

14. Grazer FM. Goldwin RM.: "Abdominoplasty assessed by a survey with emphasis on complications". Plast. Reconstr. Surg. 1977;9:257

15. Saldanha OR. De Souza Pinto EB.: "Lipoabdominoplasty with selective and safe undermining". Aesth. Plast. Surg. 2003;27(4):322.

16. Tercan M. Bekerecioglu M.: "Effects of abdominoplasty on respiratory functions: a prospective study". Ann. Plast. Surg. 2002;49(6): 617.

17. Nahas FX.: "An aesthetic classification of the abdomen based on the mioaponeurotic layer". Plast. Reconstr. Surg. 2001;108(6): 1787.

18. Van Uchelen JH. Werker PM.: "Complications of abdominoplasty in 86 patients". Plast. Reconstr. Surg. 2001; 108(2):550.

19. Netscher DT. Wigoda P. "Musculoaponeurotic plication in abdominoplasty: how durable are its effects?" Aesth. Plast. Surg. 1995;19(6):531

20. Matarasso A.: "Liposuction as an adjunct to a full abdominoplasty". Plast. Reconstr. Surg. 1995; 95(5):829.

21. Floros C. Davis PK.: "Complications and long-term results following abdominoplasty: a retrospective study”.Br J Plast. Surg. 1991 Apr; 44(3):190. 